\title{
Reports
}

\section{Malaria card: an empowering tool for patients and for epidemiological recording}

\author{
Manju Rahi ${ }^{1}$, Peeyush Mittal', Jaspreet Kaur ${ }^{2}$, Amit Sharma ${ }^{3}$ \\ ${ }^{1}$ Indian Council of Medical Research, New Delhi, India, ${ }^{2}$ ICMR- National Institute of Malaria Research, New Delhi, India, ${ }^{3}$ ICMR- National Institute of \\ Malaria Research, New Delhi, India; Molecular Medicine, International Centre for Genetic Engineering and Biotechnology, New Delhi, India \\ Keywords: malaria, epidemiological recording, community participation
}

https://doi.org/10.29392/001c.24942

\section{Journal of Global Health Reports}

Vol. 5, 2021

\begin{abstract}
Malaria places a substantial burden on the tropical and subtropical regions of the world. World Malaria Report 2020 showed that the progress in global malaria control has come to a plateau. On the other hand, India has shown considerable progress in reducing its malaria burden. Malaria surveillance system mainly depends upon health workers who are also involved with other national health programs. Community participation can play significant role in success of any control/elimination programme. Epidemiological monitoring and record keeping of disease occurrence and control programmes is important. We have designed and proposed a simple booklet called "Malaria card" which when deployed in the community, can potentially result in improved malaria tracking and record-keeping. The malaria card like immunization card for under-five children, can be utilized to capture information on important aspects of malaria and co-morbidities. It will help communities in maintaining their own record and also will give easy access to health care workers to episodes of malaria and for epidemiological tracking.
\end{abstract}

Malaria continues to be a public health problem for India. However, in recent years India has displayed remarkable decline in cases and deaths due to malaria as per World Malaria Report 2020 (WMR) from 20 million cases in 2000 to about 5.6 million in $2019 .^{1}$ The reported data by the national programme also exhibited a drop in the cases (from 2 million cases in 2000 to 0.33 million cases in 2019). ${ }^{2}$ Out of overall 0.18 million malaria cases reported in 2020, 0.11 million (63.3\%) were due to Plasmodium falciparum and more than 66000 (36.7\%) were Plasmodium vivax malaria. ${ }^{3}$ In India, malaria distribution is heterogenous and disproportionately present in under-served, remote, tribal, forested, inaccessible and backward geographical areas in malaria endemic states. In addition to higher receptivity and vulnerability, these places also suffer from sparsely present and frail healthcare system. In high transmission areas, multiple episodes of malaria in the same individual are common especially in young children and pregnant mothers due to partial immunity and increased susceptibility. ${ }^{4,5}$

The cadres of healthcare workforce like primary care workers (ie. auxiliary nurse midwife (ANM) and multipurpose workers (MPW)), community healthcare workers (HCW) (ie. Accredited Social Health Activist (ASHA), Anganwadi workers (AWW), and health volunteers are trained differentially and equipped in a manner appropriate to their expertise and involvement in the community to suspect, detect, treat and report malaria cases. ${ }^{6}$ Surveillance system continues to be paper-based system, with multiple forms and monthly aggregated data being added up from sub-center to Primary Health Centre to block level and ultimately to district which is the administrative unit for malaria control operations. ASHA and AWW being grassroot workers are entrusted with many responsibilities of other national programmes for example mother and child health services. Thus, it is impractical to expect them to recollect or carry records on her person of each and every case of malaria occurring in the community while making home visits. Also, malaria affected, poorly educated and illiterate communities are oblivious to details of malaria infection, diagnosis, species of parasite, treatment they are being given by HCWs or sometimes even the number of times they have suffered from malaria. This ignorance puts them at an added disadvantage and robs them of self-awareness of their health status. The lack of knowledge also prevents them from seeking healthcare elsewhere, especially if they are away from their native villages.

In order to empower the malaria affected individual and families, and aid the health care system in record keeping of the malaria cases, we propose deployment of 'malaria card' as a simple solution for health and epidemiological record keeping (Figure 1). These cards can be used for malaria as immunization cards are used for under-five children. The malaria card intends to capture important information from patients such as basic demographic details, blood group of the individual along-with co-existing health conditions that have an impact on malaria or its treatment.

The malaria card is to be filled by healthcare workers (same as immunization cards are filled by the medical personnel at the vaccination sites) who ensure validity of the data entered. Since all malaria cases are diagnosed by either rapid diagnostic tests or microscopy, these malaria data are captured by the public health system already. The malaria card can be bilingual or trilingual keeping the flexibility of inclusion of vernacular language for ease of the community. 

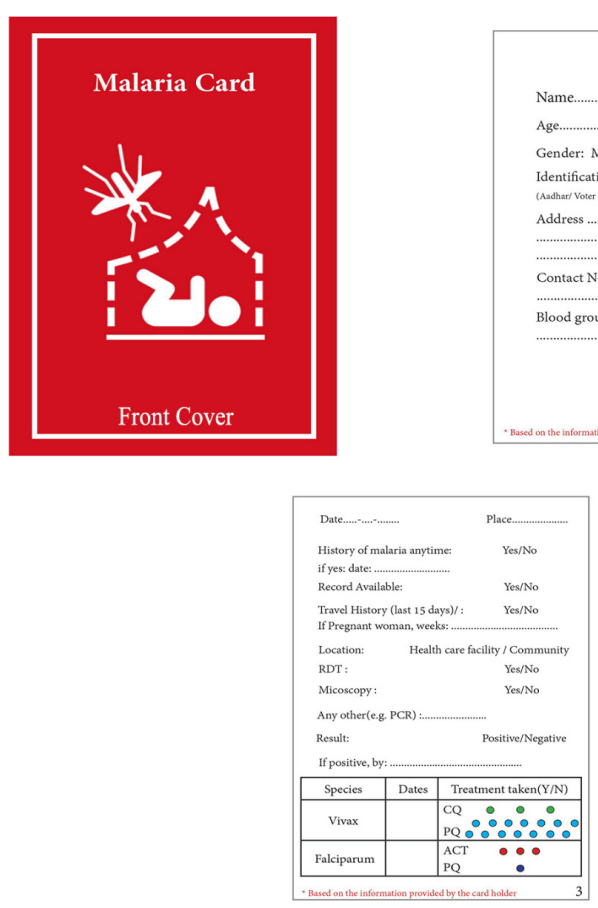
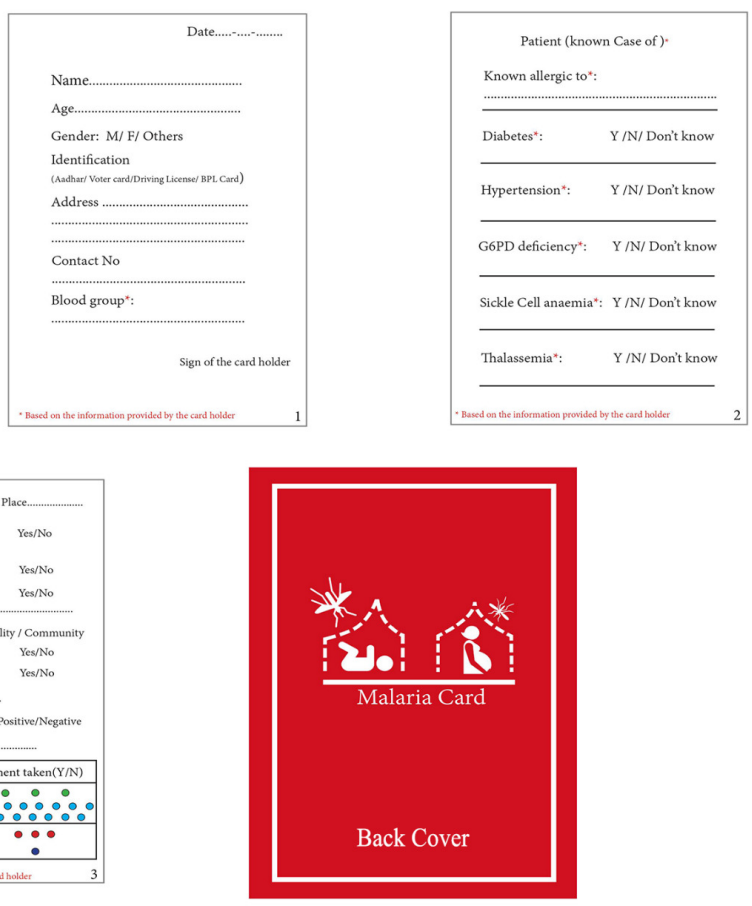

Figure 1. Malaria card

It is passport size (4.921 x $3.465 \mathrm{in})$, made of thick paper in a booklet form and will allow easy handling with minimum wear and tear. From a public health perspective, the malaria card will capture:

1. Information on recurrent episodes of malaria per patient.

2. Confirmation of malaria via diagnostic test $(\mathrm{RDT} / \mathrm{mi}-$ croscopy/PCR) and details of subsequent treatment will inform about compliance to national guidelines.

3. Information on parasite species especially Plasmodium vivax must prompt health care worker to ensure complete radical treatment with primaquine thus preventing relapses.

4. Drug compliance for both Plasmodium vivax and Plasmodium falciparum malaria cases. Treatments taken with pictorial depiction of regime completion is easy to use. Information on hemoglobinopathies and enzyme deficiency disorders like G6PD deficiency that influence choice of treatment like primaquine in the backdrop of risk of hemolysis. Documentation of this vital information would create a long lasting record that will be useful for any future antimalarial treatment and other health situations.

5. Demographic details will deduce age and sex distribution along with geographical clustering of cases.

6. Travel history will indicate threat of import/export of malaria cases.

7. Information on pregnancy status.

8. Death record.

In addition to the above, collateral benefits could also be harnessed like easy access of communities to documented proof of malaria episodes rather than relying on memory and its pitfalls. In this era of digital health records, malaria card would make its place as a parallel health record for the ease of the communities who will be empowered with their malaria information. There is also a scope of harmonization of malaria card data with the routine data of healthcare system as the same healthcare worker who is responsible for that section of community makes home visits and maintain records at the health centre. The two sets of information can be synchronized and strengthened by each other. Integrated surveillance and control operations of malaria, visceral leishmaniaisis and lymphatic filariaisis has been proposed in co-endemic areas. ${ }^{7,8}$ Immunization cards have been in use since decades in India and elsewhere and have been the backbone of maintenance of vaccination record of children with the parents. Immunization cards have been effectively implemented and contributed to control of diseases such as diphtheria-tetanus-pertussis. ${ }^{9} \mathrm{Re}-$ cently, Covid-19 immunity passport has been proposed to be incorporated in not only the health systems but also for international trave ${ }^{10}$ though its global implications and challenges are being widely discussed. ${ }^{11,12}$ Paper records such as passports and numerous other government cards are highly valuable in developing countries like India where centralized digital health records are distant yet. However, once the malaria card gets accepted and as digitization grows, the proposed malaria cards can be used to transfer their information into digital banks just as for international travel passports.

\section{CONCLUSIONS}

The malaria card can indeed serve as an integrated health record in malaria endemic regions. It has the potential to become a valuable asset to the national malaria control programme. It will also provide a sense of gravity and owner- 
ship to the communities especially in the remote and inaccessible areas of India. It can give impetus to the national malaria elimination goal and bring a sense of partnership in the community towards that goal. It will help communities in maintaining their own record and also will give easy access to health care workers to the previous episodes of malaria and will assist in epidemiological tracking. We thus believe that providing malaria cards to all malaria affected communities would be of high utility to those afflicted, for the overall healthcare system and for epidemiologists.

\section{FUNDING}

None

\section{AUTHORSHIP CONTRIBUTIONS}

AS conceptualised the idea and provided critical inputs, MR wrote the draft, PM and JK designed the card and all authors read and approved the final manuscript.

\section{COMPETING INTERESTS}

The authors completed the Unified Competing Interest form at www.icmje.org/coi_disclosure.pdf (available upon request from the corresponding author) and declare no conflicts of interest.

\section{CORRESPONDENCE TO:}

Amit Sharma

ICMR- National Institute of Malaria Research, New Delhi 110077 , India

directornimr@gmail.com

Submitted: May 28, 2021 GMT, Accepted: June 07, 2021 GMT 


\section{REFERENCES}

1. World Malaria Report 2020, Geneva. Published 2020. Accessed April 30, 2021. https://www.who.int/p ublications/i/item/9789240015791;

2. Ministry of Health and family welfare. Press release. Accessed April 30, 2021. https://www.pib.go v.in/PressReleasePage.aspx?PRID $=1677601$

3. National Vector borne disease control programme. Malaria situation in India from 2017. Accessed April 30, 2021. https://nvbdcp.gov.in/WriteReadData/1892s/ 41082350651617959011.pdf

4. Bediako Y, Adams R, Reid AJ, et al. Repeated clinical malaria episodes are associated with modification of the immune system in children. BMC Med. 2019;17:60. doi:10.1186/s12916-019-1292-y

5. Pandey M, Rahi M, Sharma A. The Indian burden of malaria in pregnancy needs assessment. In Press Cell Med. Published online 2021. doi:10.1016/i.medj.202 $\underline{1.04 .017}$

6. National Strategic Plan (2017-2022). Malaria elimination in India. Accessed April 30, 2021. http s://nvbdcp.gov.in/WriteReadData/1892s/nsp_2017-202 2.pdf
7. Rahi M, Sharma A. For malaria elimination India needs a platform for data integration. BMJ Glob Health. 2020;5(12):e004198. doi:10.1136/bmigh-202 $\underline{0-004198}$

8. Rahi M, Chaturvedi R, Das P, Sharma A. India can consider integration of three eliminable disease control programmes on malaria, lymphatic filariasis, and visceral leishmaniasis. PLoS Pathog.

2021;17:e1009492. doi:10.1371/journal.ppat.1009492

9. Wagner AL. The use and significance of vaccination cards. Hum Vaccin Immunother. 2019;15:2844-2846. d oi:10.1080/21645515.2019.1625647

10. Pavli A, Maltezou HC. COVID-19 vaccine passport for safe resumption of travel. J Travel Med. 2021;28(4). doi:10.1093/itm/taab079

11. Voigt K, Nahimana E, Rosenthal A. Flashing red lights: the global implications of COVID-19 vaccination passports. BMJ Glob Health. 2021;6:e006209. doi:10.1136/bmigh-2021-006209

12. Phelan AL. COVID-19 immunity passports and vaccination certificates: scientific, equitable, and legal challenges. Lancet. 2020;395:1595-1598. doi:1 0.1016/s0140-6736(20)31034-5 